\title{
The Prevalence of Metabolic Syndrome in Iranian Firefighters
}

\author{
Farzaneh Montazerifar (iD ${ }^{1,{ }^{*}}$, Mansour Karajibani ${ }^{2}{ }^{2}$, Razieh Hosseini ${ }^{3}$, Aliyeh Tafazzoli ${ }^{4}$ and Ahmad \\ Bolouri $^{5}$
}

${ }^{1}$ Pregnancy Health Research Center, Department of Nutrition, School of Medicine, Zahedan University of Medical Sciences, Zahedan, Iran

${ }^{2}$ Health Promotion Research Center, Department of Nutrition, School of Medicine, Zahedan University of Medical Sciences, Zahedan, Iran

${ }^{3}$ Department of Nutrition, School of Medicine, Zahedan University of Medical Sciences, Zahedan, Iran

${ }^{4}$ General Medicine, School of Medicine, Zahedan University of Medical Sciences, Zahedan, Iran

${ }^{5}$ Department of Cardiology, School of Medicine, Zahedan University of Medical Sciences, Zahedan, Iran

"Corresponding author: Associate Professor, Pregnancy Health Research Center, Department of Nutrition, School of Medicine, Zahedan University of Medical Sciences, Zahedan, Iran. Email: fmontazerifar@gmail.com

Received 2020 February 19; Revised 2020 May 25; Accepted 2020 May 26.

\begin{abstract}
Background: Metabolic syndrome (MetS) is closely related to the risk of cardiovascular disease(CVD), particularly in stressful occupations. Firefighting is a hazardous profession and is associated with high metabolic risk. However, limited studies on firefighters are available.

Objectives: This study aimed to determine the prevalence of MetS among firefighters in Zahedan city, Iran.

Methods: This cross-sectional study was performed on 140 firefighters (age range, 16 - 55 years) employed at the fire department in Zahedan city, southeast Iran. Anthropometric characteristics and metabolic risk parameters, including blood pressure (BP), fasting blood sugar (FBS), and lipid profile were measured, and the prevalence of metabolic syndrome was assessed based on National Cholesterol Education Program Adult Treatment Panel III (NCEP ATP III) criteria.

Results: MetS was presented in 47 (33.6\%) of the firefighters. Among the metabolic risk factors, abdominal obesity (87.1\%), low-high density lipoprotein (HDL-C) (62.1\%), and hypertriglyceridemia (61.4\%) were more prevalent. Moreover, a significant increasing trend was found with increasing age and work experience (both, $\mathrm{P}=0.05$ ). However, no significant differences were found for the remaining metabolic risk factors.

Conclusions: The findings showed that about one-third of the firefighters had MetS. Age, abdominal obesity, and dyslipidemia were independent risk factors for MS in the firefighters. Thus, efforts should be undertaken to implement healthy lifestyle promotion programs for firefighters.
\end{abstract}

Keywords: Metabolic Syndrome, Firefighters, Iran

\section{Background}

Metabolic syndrome (MetS) is a global epidemic disorder associated with pathological conditions, which is characterized by dyslipidemia [high triglyceride and low HDL cholesterol (HDL-C)], abdominal obesity, high fasting blood sugar (FBS), and hypertension (1). It is predicted that by 2025 , around 300 million people worldwide will have type 2 diabetes, which is one of the major risk factors for metabolic syndrome (2). The prevalence of MetS is more common in some developing countries than in Western countries (1). MetS is also prevalent in Iran and has a growing trend with age in both sexes $(3,4)$. It has been reported that the syndrome is closely related to the risk of cardiovascular disease and diabetes (5).

The inherent causes of MetS are not well-known; however, factors such as aging, stressful occupations, sedentary activity, and genetics indirectly influence it (6). Work stress is one of the major risk factors for metabolic syndrome. A high prevalence of hypertension, hyperlipidemia, diabetes, and other cardiovascular disease (CVD) risk factors have been reported in stressful occupations such as emergency services, police work, and firefighting (7). Firefighting has a high metabolic burden and often results in severe cardiovascular stress $(8,9)$. The prevalence of MetS has been confirmed in $14 \%$ of German firefighters (2) and 56.6\% of Iranian firefighters (10). Almost $45 \%$ of all work-related deaths among firefighters are reported to be caused by CVD (9). On-duty cardiac events frequently occur among firefighters prone to underlying structural heart disease. Intense physical activity, emotional stress, and pollutants present in the fire are the major risk factors of sudden cardiac death in the firefighting occupation (11). Central obesity as one of the metabolic syndrome components is a 
strong risk factor for diabetes and CVD, which has been reported in $45.9 \%$ of the US firefighters (9) and $28.9 \%$ of Iranian firefighters (10). Chronic stressors such as lone-term shift work and irregular mealtimes may increase obesity, hypertension, and dyslipidemia in firefighters. These factors identified as metabolic syndrome components may increase CVD risk (9). According to the literature review, few studies have evaluated the prevalence of MetS and its components in Iranian firefighters.

\section{Objectives}

This study was performed to investigate the prevalence of metabolic syndrome in firefighters of Zahedan city, southeast Iran.

\section{Methods}

In this cross-sectional study, 140 male firefighters (mean age, $36.8 \pm 7.5$ years; range, 16 - 55 years) from the Fire Department of Zahedan were evaluated between June to October 2017. The inclusion criteria were cigarette smoking and at least two years of full-time employment as a firefighter with no history of underlying diseases including hypertension, kidney failure, hypo/hyperthyroidism, and liver diseases, known cardiovascular disease, and diabetes mellitus. The protocol of the study was approved by the Ethics Committee of Zahedan University of Medical Sciences (Code IR.ZAUMS.REC. 1396.2). Informed consent was also obtained from all the participants. Then, a personal data questionnaire on demographic characteristics, including age, education level, smoking status, work experience, and medical history, was completed for all the subjects.

Bodyweight and height were measured with light clothing and without shoes using a platform scale and a fixed stadiometer, respectively. The body mass index (BMI) was calculated as weight $(\mathrm{kg})$ divided by height squared $\left(\mathrm{m}^{2}\right)$. Waist circumstance was measured using a nonelastic tape at the narrowest point between the lower edge of the rib and the upper iliac crest. Blood pressure was measured twice using a standard sphygmomanometer on the right arm in a sitting position after resting for 10 minutes. The mean of the two blood pressure readings was used in the analysis.

A venous fasting blood sample was obtained to measure biochemical parameters. Fasting blood sugar (FBS) and serum lipid profile (total cholesterol, triglyceride, LDL$\mathrm{C}$, and HDLC) levels were evaluated using the enzymatic calorimetric method with the Pars Azmun Kit (Tehran, Iran). Metabolic syndrome was diagnosed based on the
National Cholesterol Education Program, Adult Treatment Panel III, 2005 (NCEP/ATP III) criteria (1). According to this approach, the presence of three or more of the following components was required to detect MetS: Abdominal obesity (waist circumference $>102 \mathrm{~cm}$ in men), high blood pressure (systolic blood pressure $>130 \mathrm{~mm} \mathrm{Hg}$ and diastolic blood pressure $>85 \mathrm{~mm} \mathrm{Hg}$ ) or current use of antihypertensive medication, increased triglyceride levels (> $150 \mathrm{mg} / \mathrm{dL}$ ) or drug treatment for high triglycerides, decreased HDL-C levels ( $<40 \mathrm{mg} / \mathrm{dL}$ in men), and elevated fasting blood sugar $(>100 \mathrm{mg} / \mathrm{dL})$ or drug treatment for elevated FBS.

\subsection{Statistical Analysis}

The data were analyzed using SPSS software version 21 (SPSS, Inc., Chicago, IL, USA). The anthropometric and biochemical parameters were presented as mean \pm standard deviation (SD) and frequency (\%). A student's t-test and a chi-square test were used to compare the quantitative variables and the categorical variables, respectively. Moreover, a Pearson correlation test was used to determine associations between the continuous variables. A P value of $<0.05$ was considered significant.

\section{Results}

The demographic, anthropometric, and clinical characteristics of the firefighters are presented in Table 1 . The mean age of the subjects was $36.8 \pm 7.5$ years (range, 16 $55)$, and the average work experience was $12.4 \pm 0.63$ years (range, 2 - 30). A significant difference was found between the two groups in terms of age and work experience (both, $\mathrm{P}=0.05$ ).

The firefighters with MetS had higher weight and BMI than those without MetS $(\mathrm{P}<0.0001)$. Moreover, the firefighters with Mets had significantly higher values of waist circumference (WC), blood pressure, triglyceride ( $P$ $<0.0001)$, and total cholesterol $(\mathrm{P}<0.001)$, but significantly lower HDL-C levels $(\mathrm{P}<0.0001)$ compared to the other group.

As shown in Table 2, MetS was detected in almost one out of four firefighters (33.6\%). The highest prevalence was shown in the age group $>30$ years (85.1\%) and in those with work experience over 10 years (69.9\%).

Table 3 outlines the prevalence of the normal or abnormal values of the metabolic risk factors in the firefighters according to the NCEP/ATP III criteria. Among the metabolic risk factors, the highest prevalence with abnormal values was observed in high WC (87.1\%), low HDL-C levels (62.1\%), and hypertriglyceridemia (61.4\%).

The Pearson correlation test showed a significant positive association between age triglyceride $(\mathrm{r}=0.318, \mathrm{P}<$ 


\begin{tabular}{|c|c|c|c|}
\hline Variables & $\operatorname{MetS}(N=47)$ & No MetS $(\mathrm{N}=93)$ & PValue $^{\text {b }}$ \\
\hline Age (years) & $38.1 \pm 7.6$ & $32.6 \pm 6.9$ & 0.05 \\
\hline $\begin{array}{l}\text { Work experience } \\
\text { (years) }\end{array}$ & $13.9 \pm 1.2$ & $10.6 \pm 0.73$ & 0.05 \\
\hline Weight (kg) & $84.6 \pm 10.7$ & $74.1 \pm 9.8$ & $<0.0001$ \\
\hline Height $(\mathrm{cm})$ & $175.8 \pm 5.2$ & $175.6 \pm 5.8$ & 0.84 \\
\hline BMI $\left(\mathrm{kg} / \mathrm{m}^{2}\right)$ & $27.4 \pm 3.7$ & $24.0 \pm 3.1$ & $<0.0001$ \\
\hline $\mathrm{WC}(\mathbf{c m})$ & $96.3 \pm 8.2$ & $87.7 \pm 6.6$ & $<0.0001$ \\
\hline SBP (mmHg) & $138.0 \pm 5.7$ & $118.2 \pm 5.3$ & $<0.0001$ \\
\hline DBP (mmHg) & $90 \pm 3.0$ & $78.4 \pm 4.0$ & $<0.0001$ \\
\hline FBS $(\mathrm{mg} / \mathrm{dL})$ & $91.0 \pm 27.9$ & $84.0 \pm 7.6$ & 0.1 \\
\hline $\mathrm{TC}(\mathrm{mg} / \mathrm{dL})$ & $201.3 \pm 46.7$ & $173.9 \pm 34.2$ & $<0.001$ \\
\hline Triglyceride (mg/dL) & $228.5 \pm 91.4$ & $96.9 \pm 36.7$ & $<0.0001$ \\
\hline LDL-C (mg/dL) & $121.3 \pm 44.5$ & $109.1 \pm 28.8$ & 0.055 \\
\hline HDL-C (mg/dL) & $30.6 \pm 4.2$ & $44.8 \pm 9.3$ & $<0.0001$ \\
\hline
\end{tabular}

Abbreviations: BMI, body mass index; DBP, diastolic blood pressure; FBS, fasting glucose blood sugar; HDL-C, high density lipoprotein cholesterol; LDL-C, low density lipoprotein cholesterol; MetS, metabolic syndrome; SBP, systolic blood pressure; TC, cholesterol; WC, waist-circumference.

${ }^{a}$ Data are presented as mean \pm standard deviation.

${ }^{\mathrm{b}}$ Comparison was performed between the MetS and no MetS groups using the student $t$-test.

\begin{tabular}{|c|c|c|c|}
\hline \multirow{2}{*}{ Variables } & \multicolumn{2}{|c|}{ Groups } & \multirow{2}{*}{ PValue $^{b}$} \\
\hline & MetS & No MetS & \\
\hline Firefighters & $47(33.6)$ & $93(66.4)$ & $<0.0001$ \\
\hline Age (years) & & & 0.05 \\
\hline$<30$ & $7(14.9)$ & $28(30.1)$ & \\
\hline$>30$ & $40(85.1)$ & $65(69.9)$ & \\
\hline $\begin{array}{l}\text { Work experience } \\
\text { (years) }\end{array}$ & & & 0.05 \\
\hline$<10$ & $15(31.9)$ & $50(53.8)$ & \\
\hline$>10$ & $32(68.1)$ & $43(46.2)$ & \\
\hline
\end{tabular}

Abbreviations: MetS, metabolic syndrome.

${ }^{\mathrm{a}}$ Data are presented as number $(\%)$.

${ }^{\mathrm{b}}$ Comparison was performed between the normal and abnormal values using the chi-square test.

$0.01)$ and blood pressure $(r=0.385, P=0.008)$, and also, between WC and triglyceride $(\mathrm{r}=0.518, \mathrm{P}<0.0001)$. Further, a significant negative association was found between age and WC with HDL-C $(\mathrm{r}=-0.604, \mathrm{P}=0.035$; and $\mathrm{r}=-0.404$, $\mathrm{P}$ $=0.005)$, respectively.
Table 3. Prevalence of the Normal or Abnormal Values of Metabolic Risk Factors in the Firefighters According to the NCEP/ATP III Criteria ${ }^{a}$

\begin{tabular}{lccc}
\hline \multirow{2}{*}{ Variables } & \multicolumn{2}{c}{ Groups } & PValue $^{\mathbf{b}}$ \\
& Normal & No Normal & \\
\hline BMI $\left(\mathbf{k g} / \mathbf{m}^{2}\right)$ & $80(57.1)$ & $60(42.9)$ & 0.17 \\
WC $(\mathbf{c m})$ & $18(12.9)$ & $122(87.1)$ & $<0.0001$ \\
\hline BP $(\mathbf{m m H g})$ & $77(55)$ & $63(45)$ & 0.0 \\
\hline FBS $(\mathbf{m g} / \mathbf{d L})$ & $81(57.8)$ & $59(42.2)$ & 0.15 \\
\hline Triglyceride $(\mathbf{m g} / \mathbf{d L})$ & $54(38.6)$ & $86(61.4)$ & $<0.001$ \\
\hline HDL-C $(\mathbf{m g} / \mathbf{d L})$ & $53(37.9)$ & $87(62.1)$ & $<0.001$ \\
\hline
\end{tabular}

Abbreviations: BMI, body mass index; BP, blood pressure; FBS, fasting glucose blood sugar; HDL-C, high density lipoprotein cholesterol; MetS, metabolic syndrome; WC, waist circumference.

${ }^{\mathrm{a}}$ Data are presented as number (\%).

${ }^{\mathrm{b}}$ Comparison was performed between the normal and abnormal values using the chi-square test.

\section{Discussion}

This cross-sectional study is one of the few studies conducted on firefighters. The prevalence of MetS and its components was investigated in a total of 140 male firefighters working at the Fire Department, Zahedan, southeast Iran. The NCEP/ATP III criteria were used to determine MetS, and its overall prevalence was $33.6 \%$ in the firefighters.

Studies evaluating the role of occupational exposure have shown that firefighters experience high metabolic burden $(8,9)$, which might, if repeated and prolonged, cause cardiovascular disease (8). A study reported that the risk of MetS in firefighters was not significantly different from that in most other occupational people (12). However, few studies have been carried out on the prevalence of MetS in Iranian firefighters, and most of the studies have been performed on general population or patients. The prevalence of metabolic syndrome in the adult urban population in Iran was found between $18.3 \%$ - 57.8\% (13). However, studies performed on firefighters in Iran and other countries demonstrated that the prevalence of MetS among firefighters was $28.9 \%$ in Iran, $39 \%$ in the United States (9\% in Colorado) (8, 14), 28.3\% in Cyprus (15), 21.4\% in Korean Career Firefighters (12), and 14.4\% in Germany (2). The observed differences in the prevalence of MetS in different communities and specific occupational conditions vary according to the criteria used to define it (2) and based on lifestyle-related factors, age, gender, excessive physical exertion, occupational stress levels and (shift work (1, 7, $12,16)$. Overall, some previously published studies showed that the rate of metabolic risk factors including obesity, hypertention, and dyslipidemia was higher in firefighters compared to the general population $(8,12)$. However, Strauss et al. (2). in their study reported that metabolic risk 
factors did not significantly differ between office workers and firefighters.

There is evidence that obesity is related to a high risk of occupational disability in specific job groups (17). The association between BMI and metabolic syndrome in firefighters was reported in several studies $(9,17,18)$. In our study, BMI in subjects with MetS was above the normal range of $\leq 25 \mathrm{~kg} / \mathrm{m}^{2}$, and thus, it was consistent with overweight. Moreover, $42.9 \%$ of the firefighters in our study were overweight, which is above the prevalence of $19 \%-39.7 \%$ among firefighters reported in some previous studies $(17,18)$. In other cross-sectional studies in the United States and the UK, overweight and obesity was prevalent in more than three-quarters of firefighters $(8,19)$. However, other studies on American firefighters showed a higher prevalence of overweight and obesity $(8,9,20)$. The reason might be the fact that the evaluation of high muscle mass and low body fat percentage in most firefighters using BMI as a classification tool is not correct (9). WC is one of the indices of central obesity and estimates the risk of chronic obesityrelated diseases, including metabolic syndrome. It is one of the most serious risk factors for cardiovascular disease (10). In the present study, an increased WC was shown in $87.1 \%$ of the firefighters. Smith et al. (9) and Strauss et al. (2) reported that central obesity was detected in $45.9 \%$ and $32 \%$ of firefighters, respectively. Although increased WC is likely related to sedentary activities, other lifestyle-related factors, including dietary intakes (21) and professional activities, also affect abdominal obesity (2), which we did not assess in our study.

MetS is a complex metabolic disorder, which is associated with high risk of cardiovascular disease (2). According to our findings, metabolic syndrome and obesity, particularly abdominal obesity, are considered as two potential risk factors for the occurrence of cardiovascular disease (2). The study of the other risk factors also revealed that the mean systolic and diastolic blood pressure, serum levels, and total cholesterol and triglyceride were significantly higher, but the HDL-C levels were significantly lower, in firefighters with MetS compared with those without MetS. In addition, the prevalence of the normal or abnormal values of the metabolic risk factors in the firefighters according to the NCEP/ATP III criteria in our study revealed that high WC, low HDL-C, and hypertriglyceridemia were the most common metabolic risk factors in the male firefighters. In a systematic review and meta-analysis, the most prevalent components of MetS in the general population were low HDL-C and hypertriglyceridemia (4). A previous study of police officers demonstrated that workrelated stress through dyslipidemia (hypertriglyceridemia and low HDL-C) induced MetS (7) and was confirmed as an important underlying factor in cardiac events (8). A signif- icant positive association between WC and triglyceride, as well as an adverse association between WC and HDL-C in our study, suggested that these factors may predict cardiovascular disease in the future.

In agreement with a previous study (14), a significant increase trend was shown by age groups in our study, as MetS increased from $14.9 \%$ in the group below 30 years to $85.1 \%$ in the group over 30 years. A negative association between age and HDL-C, as well as a positive association between triglyceride and blood pressure, suggested that increasing age was independently associated with cardiovascular disease. Lee and Kim (12) demonstrated that age and obesity were independent risk factors for metabolic syndrome in both firefighters and general workers.

Another result from our study was that work experience was effective in developing metabolic syndrome, as the prevalence of MetS increased in firefighters with work experience above 10 years. There is evidence that stressful conditions such as hard physical activity, impaired sleep rhythm, emotional stress, and environmental pollutants in occupations such as firefighting can cause cardiac events (11).

Our study has several limitations: The first limitation is that our survey was restricted to firefighting, and no other occupational groups were evaluated and compared.

Second, we did not collect data on the diet, physical activity, and shift the work of the participants. Therefore, given the cross-sectional design of the study, we could not discuss the causation of obesity, particularly abdominal obesity.

Further studies are recommended to compare the prevalence of metabolic syndrome and CVD risk factors among different occupational groups, especially stressful jobs.

\subsection{Conclusion}

The findings showed that about one-third of the firefighters had MetS. Age, abdominal obesity, and dyslipidemia were independent risk factors for MetS in the firefighters, which may predict the incidence of cardiovascular disease in the future. Thus, efforts should be undertaken on the implementation of healthy lifestyle promotion programs for firefighters.

\section{Footnotes}

Authors' Contribution: Farzaneh Montazerifar and Mansour Karajibani were equally involved in the writing of the manuscript and experimental design. Farzaneh Montazerifar and Razieh Hosseini drafted the paper. Alieh Tafazzoli collected data. Ahmad Bolouri assessed biochemical tests 
and data interpretation. Farzaneh Montazerifar and Mansour Karajibani analyzed the data. Farzaneh Montazerifar and Mansour Karajibani reviewed the draft. All the authors read and approved the final manuscript.

Conflict of Interests: The authors have no competing interests.

Ethical Approval: The protocol of the study was approved by the Ethics Committee of the Zahedan University of Medical Sciences (Code IR.ZAUMS.REC. 1396.2).

Funding/Support: This study was funded by the Research Deputy of the Zahedan University of Medical Sciences, Zahedan, Iran (Grant number 8116).

Informed Consent: Informed consent forms were obtained from all the participants.

\section{References}

1. Saklayen MG. The Global Epidemic of the Metabolic Syndrome. Curr Hypertens Rep. 2018;20(2):12. doi: 10.1007/s11906-018-0812-z. [PubMed: 29480368]. [PubMed Central: PMC5866840].

2. Strauss M, Foshag P, Przybylek B, Horlitz M, Lucia A, Sanchis-Gomar F, et al. Occupation and metabolic syndrome: is there correlation? A cross sectional study in different work activity occupations of German firefighters and office workers. Diabetol Metab Syndr. 2016;8(1):57. doi: 10.1186/s13098-016-0174-0. [PubMed: 27555885]. [PubMed Central: PMC4994412].

3. Ferns GA, Ghayour-Mobarhan M. Metabolic syndrome in Iran: A review. Translational Metabolic Syndrome Research. 2018;1:10-22. doi: 10.1016/j.tmsr.2018.04.001.

4. Mazloomzadeh S, Rashidi Khazaghi Z, Mousavinasab N. The Prevalence of Metabolic Syndrome in Iran: A Systematic Review and Metaanalysis. Iran J Public Health. 2018;47(4):473-80. [PubMed: 29900131]. [PubMed Central: PMC5996331].

5. Li K, Ochoa E, Lipsey T, Nelson T. Correlates of atherosclerotic cardiovascular disease risk in older Colorado firefighters. Occup Med (Lond). 2018;68(1):51-5. doi: 10.1093/occmed/kqx192. [PubMed: 29351620].

6. Kaur J. A comprehensive review on metabolic syndrome. Cardiol Res Pract. 2014;2014:943162. doi: 10.1155/2014/943162. [PubMed: 24711954]. [PubMed Central: PMC3966331].

7. Garbarino S, Magnavita N. Work Stress and Metabolic Syndrome in Police Officers. A Prospective Study. PLoS One. 2015;10(12). e0144318. doi: 10.1371/journal.pone.0144318. [PubMed: 26641879]. [PubMed Central: PMC4671563].

8. Byczek L, Walton SM, Conrad KM, Reichelt PA, Samo DG. Cardiovascular risks in firefighters: implications for occupational health nurse practice. AAOHNJ. 2004;52(2):66-76. [PubMed: 14979617].
9. Smith DL, Fehling PC, Frisch A, Haller JM, Winke M, Dailey MW. The prevalence of cardiovascular disease risk factors and obesity in firefighters. J Obes. 2012;2012:908267. doi: 10.1155/2012/908267. [PubMed: 22888409]. [PubMed Central: PMC3409612].

10. Ebrahimi-Mameghani M, Farsad Naimi AR, Nourmohammadi M, Azabdaftari N. [Prevalence of Metabolic syndrome in Tabriz city firefighters]. J Kermanshah Univ Med Sci. 2011;15(4). Persian. e79310.

11. Smith DL, Barr DA, Kales SN. Extreme sacrifice: sudden cardiac death in the US Fire Service. Extrem Physiol Med. 2013;2(1):6. doi: 10.1186/20467648-2-6. [PubMed: 23849605]. [PubMed Central: PMC3710100].

12. Lee W, Kim J. Prevalence of Metabolic Syndrome and Related Factors in Korean Career Firefighters in Comparisons With Other Occupational Groups. J Occup Environ Med. 2017;59(4):384-8. doi: 10.1097/JOM.0000000000000956. [PubMed: 28157765].

13. Kalan Farmanfarma K, Kaykhaei MA, Adineh HA, Mohammadi M, Dabiri S, Ansari-Moghaddam A. Prevalence of metabolic syndrome in Iran: A meta-analysis of 69 studies. Diabetes Metab Syndr. 2019;13(1):792-9. doi: 10.1016/j.dsx.2018.11.055. [PubMed: 30641809].

14. Li K, Lipsey T, Leach HJ, Nelson TL. Cardiac health and fitness of Colorado male/female firefighters. Occup Med (Lond). 2017;67(4):268-73. doi: 10.1093/occmed/kqx033. [PubMed: 28340191].

15. Baur DM, Christophi CA, Kales SN. Metabolic syndrome is inversely related to cardiorespiratory fitness in male career firefighters. J Strength Cond Res. 2012;26(9):2331-7. doi: 10.1519/JSC.ob013e31823e9b19. [PubMed: 22067249].

16. Choi B, Ko S, Kojaku S. Resting heart rate, heart rate reserve, and metabolic syndrome in professional firefighters: A cross-sectional study. Am J Ind Med. 2017;60(10):900-10. doi: 10.1002/ajim.22752. [PubMed: 28869309].

17. Soteriades ES, Hauser R, Kawachi I, Christiani DC, Kales SN. Obesity and risk of job disability in male firefighters. Occup Med (Lond). 2008;58(4):245-50. doi: 10.1093/occmed/kqm153. [PubMed: 18204003].

18. Poston WS, Haddock CK, Jahnke SA, Jitnarin N, Tuley BC, Kales SN. The prevalence of overweight, obesity, and substandard fitness in a population-based firefighter cohort. J Occup Environ Med. 2011;53(3):266-73. doi: 10.1097/JOM.0b013e31820af362. [PubMed: 21386691]. [PubMed Central: PMC5826653].

19. Yoo HL, Franke WD. Prevalence of cardiovascular disease risk factors in volunteer firefighters. J Occup Environ Med. 2009;51(8):958-62. doi: 10.1097/JOM.ob013e3181af3a58. [PubMed: 19620889].

20. Wilkinson ML, Brown AL, Poston WS, Haddock CK, Jahnke SA, Day RS. Physician weight recommendations for overweight and obese firefighters, United States, 2011-2012. Prev Chronic Dis. 2014;11. E116. doi: 10.5888/pcd11.140091. [PubMed: 25010998]. [PubMed Central: PMC4093977].

21. Yang J, Farioli A, Korre M, Kales SN. Dietary Preferences and Nutritional Information Needs Among Career Firefighters in the United States. Glob Adv Health Med. 2015;4(4):16-23. doi: 10.7453/gahmj.2015.050. [PubMed: 26331100]. [PubMed Central: PMC4533657] 